\title{
Revised Structures of Epohelmins A and B Isolated as Lanosterol Synthase Inhibitors from a Fungal Strain FKI-0929
}

\author{
Masaaki Shibuya, Barry B. Snider, Yuichi Sakano, Hiroshi Tomoda, Satoshi Ōmura, \\ Yutaka Ebizuka
}

Received September 7, 2005 / Accepted September 20, 2005

(C) Japan Antibiotics Research Association

\begin{abstract}
The structures of epohelmins A and B isolated as lanosterol synthase inhibitors from a fungal strain FKI0929 were revised to be $1 \alpha$-hydroxy-3 $\alpha$-(4'-oxoundec(5'E)-enyl)-pyrrolizidine and $1 \beta$-hydroxy- $3 \alpha-\left(4^{\prime}-\right.$ oxoundec-(5'E)-enyl)-pyrrolizidine, respectively, by comparison with spectral data of synthetic compounds.
\end{abstract}

Keywords epohelmin A, epohelmin B, 1-hydroxy-3 $\alpha$ (4'-oxoundec-( $\left.5^{\prime} E\right)$-enyl)-pyrrolizidine, cholesterol biosynthesis, lanosterol synthase
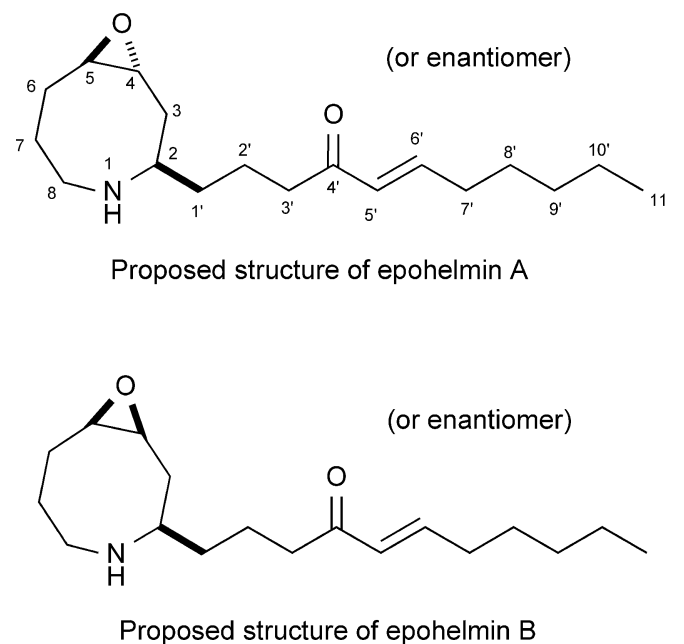

Fig. 1 Structures of epohelmins A and B.
In screening for recombinant human lanosterol synthase inhibitors from microbes, lanopylins [1] and epohelmins [2] were isolated from an actinomycete strain, Streptomyces sp. K99-5041, and a fungal strain FKI-0929, respectively.

The structures of lanopylins $\mathrm{A}_{1}$ and $\mathrm{B}_{1}$ were assigned as (3E)-isohexadecylmethylidene-2-methyl-1-pyrroline and (3E)-hexadecylmethylidene-2-methyl-1-pyrroline, respectively, by spectroscopic analyses [1]. In a recent

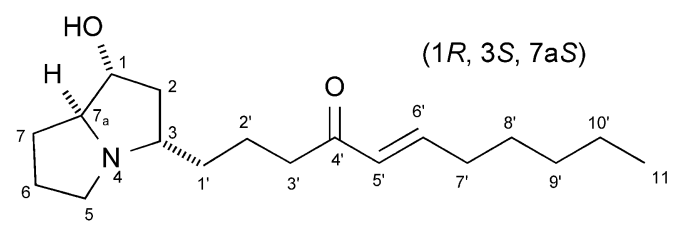

Revised structure of epohelmin A

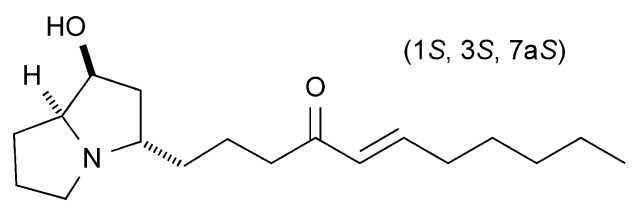

Revised structure of epohelmin B
M. Shibuya (Corresponding author), Y. Sakano, Y. Ebizuka: Graduate School of Pharmaceutical Sciences, The University of Tokyo, 7-3-1 Hongo, Bunkyo-ku, Tokyo 113-0033, Japan, E-mail: shibuyam@mol.f.u-tokyo.ac.jp
B. B. Snider: Department of Chemistry MS015, Brandeis University, Waltham, Massachusetts 02454-9110, USA

H. Tomoda, S. Ōmura: Kitasato Institute for Life Sciences, Kitasato University, and The Kitasato Institute, 5-9-1 Shirokane, Minato-ku, Tokyo 108-8641, Japan 
Table 1 NMR assignment for the acetate salts of epohelmins $A$ and $B$

\begin{tabular}{|c|c|c|c|c|c|}
\hline \multirow[b]{2}{*}{ Position } & \multicolumn{2}{|r|}{ Epohelmin A } & \multirow[b]{2}{*}{ Position } & \multicolumn{2}{|r|}{ Epohelmin B } \\
\hline & ${ }^{13} \mathrm{C} \delta \mathrm{ppm}$ & ${ }^{1} \mathrm{H} \delta \mathrm{ppm}(\mathrm{J}$ in $\mathrm{Hz})$ & & ${ }^{13} \mathrm{C} \delta \mathrm{ppm}$ & ${ }^{1} \mathrm{H} \delta \mathrm{ppm}(J$ in $\mathrm{Hz})$ \\
\hline 1 & 74.3 & $4.17(1 \mathrm{H}, \mathrm{td}, 6.3,3.0)$ & 1 & 70.2 & $4.38(1 \mathrm{H}, \mathrm{br} \mathrm{s})$ \\
\hline \multirow[t]{2}{*}{2} & 40.9 & $2.06(1 \mathrm{H}, \mathrm{m})$ & 2 & 42.3 & $2.22(1 \mathrm{H}, \mathrm{m})$ \\
\hline & & $2.60(1 \mathrm{H}, \mathrm{m})$ & & & $2.10(1 \mathrm{H}, \mathrm{m})$ \\
\hline 3 & 67.3 & $2.99(1 \mathrm{H}, \mathrm{m})$ & 3 & 66.7 & $3.30(1 \mathrm{H}, \mathrm{m})$ \\
\hline 4 & & & 4 & & \\
\hline \multirow[t]{2}{*}{5} & 51.9 & $3.43(1 \mathrm{H}, \mathrm{dt}, 12.0,7.8)$ & 5 & 52.9 & $3.50(1 \mathrm{H}, \mathrm{m})$ \\
\hline & & $2.96(1 \mathrm{H}, \mathrm{dt}, 12.0,6.0)$ & & & $2.88(1 \mathrm{H}, \mathrm{dt}, 11.0,6.0)$ \\
\hline 6 & 24.2 & $2.04(2 \mathrm{H}, \mathrm{m})$ & 6 & 26.8 & $2.04(2 \mathrm{H}, \mathrm{m})$ \\
\hline \multirow[t]{2}{*}{7} & 28.4 & $2.26(1 \mathrm{H}, \mathrm{m})$ & 7 & 24.1 & $2.21(1 \mathrm{H}, \mathrm{m})$ \\
\hline & & $1.72(1 \mathrm{H}, \mathrm{m})$ & & & $1.88(1 \mathrm{H}, \mathrm{m})$ \\
\hline $7 a$ & 73.9 & $4.11(1 \mathrm{H}, \mathrm{td}, 8.3,3.3)$ & $7 a$ & 70.8 & $4.47(1 \mathrm{H}, \mathrm{br} d t, 8.5,4.3)$ \\
\hline \multirow[t]{2}{*}{$1^{\prime}$} & 31.2 & $1.92(1 \mathrm{H}, \mathrm{m})$ & $1^{\prime}$ & 30.4 & $1.96(1 \mathrm{H}, \mathrm{m})$ \\
\hline & & $1.82(1 \mathrm{H}, \mathrm{m})$ & & & $1.81(1 \mathrm{H}, \mathrm{m})$ \\
\hline $2^{\prime}$ & 21.1 & $1.64(2 \mathrm{H}, \mathrm{ddt}, 7.7,7.7,7.7)$ & $2^{\prime}$ & 21.3 & $1.65(2 \mathrm{H}, \mathrm{ddt}, 7.5,7.5,7.5)$ \\
\hline $3^{\prime}$ & 39.1 & $2.60(2 \mathrm{H}, \mathrm{t}, 7.0)$ & $3^{\prime}$ & 39.3 & $2.61(2 \mathrm{H}, \mathrm{td}, 7.1,2.1)$ \\
\hline $4^{\prime}$ & 199.6 & & $4^{\prime}$ & 199.8 & \\
\hline $5^{\prime}$ & 130.1 & $6.07(1 \mathrm{H}, \mathrm{dt}, 16.0,1.5)$ & $5^{\prime}$ & 130.2 & $6.07(1 \mathrm{H}, \mathrm{dt}, 15.5,1.5)$ \\
\hline $6^{\prime}$ & 148.2 & $6.83(1 \mathrm{H}, \mathrm{dt}, 15.7,6.9)$ & $6^{\prime}$ & 148.1 & $6.84(1 \mathrm{H}, \mathrm{dt}, 16.0,7.0)$ \\
\hline $7^{\prime}$ & 32.5 & $2.21(2 \mathrm{H}, \mathrm{tdd}, 7.0,7.0,1.5)$ & $7^{\prime}$ & 32.5 & $2.21(2 \mathrm{H}, \mathrm{tdd}, 7.0,7.0,1.5)$ \\
\hline $8^{\prime}$ & 27.7 & $1.47(2 \mathrm{H}, \mathrm{tt}, 7.4,7.4)$ & $8^{\prime}$ & 27.7 & $1.46(2 \mathrm{H}, \mathrm{tt}, 7.4,7.4)$ \\
\hline $9^{\prime}$ & 31.3 & $1.31(2 \mathrm{H}, \mathrm{m})$ & $9^{\prime}$ & 31.3 & $1.30(2 \mathrm{H}, \mathrm{m})$ \\
\hline $10^{\prime}$ & 22.4 & $1.29(2 \mathrm{H}, \mathrm{m})$ & $10^{\prime}$ & 22.4 & $1.32(2 \mathrm{H}, \mathrm{m})$ \\
\hline $11^{\prime}$ & 13.9 & $0.90(3 \mathrm{H}, \mathrm{t}, 7.0)$ & $11^{\prime}$ & 13.9 & $0.89(3 \mathrm{H}, \mathrm{t}, 7.0)$ \\
\hline
\end{tabular}

${ }^{1} \mathrm{H}(500.00 \mathrm{MHz})$ and ${ }^{13} \mathrm{C}(125.65 \mathrm{MHz}) \mathrm{NMR}$ spectra were obtained in chloroform- $d$. Epohelmin A contains $0.85 \sim 0.90$ equiv of $\mathrm{HOAC}$, while epohelmin B contains 1 equiv of HOAc. The acetate absorbs at $\delta 2.00 \sim 2.01$ in the ${ }^{1} \mathrm{H}$ NMR spectra and $\delta 176.5 \sim 177$ and $\delta 22.5 \sim 23$ in the ${ }^{13} \mathrm{C}$ NMR spectra.

report, the structure of lanopylin $\mathrm{B}_{1}$ was confirmed by synthesis [3].

Epohelmins A and B were proposed to be two of the diastereomers of 4,5-epoxy-2-(4'-oxoundec-(5'E)-enyl)heptamethylenamine, and their relative stereochemical configurations to be $(2 R, 4 R, 5 R)$ or $(2 S, 4 S, 5 S)$ for epohelmin $\mathrm{A}$, and $(2 R, 4 S, 5 R)$ or $(2 S, 4 R, 5 S)$ for epohelmin B, respectively, by spectroscopic analyses [2]. However, in the process of structural determination, we did not place much importance on the evidence that 4,5-epoxyheptamethylenamines readily cyclize to 1hydroxypyrrolizidines [4,5], and that the methine hydrogens and carbons of trans and cis-epoxycylooctanes absorb at $\delta 2.8 \sim 2.9$ and $\delta 59.6 \sim 55.6$, respectively [6]. Recently, $1 \alpha$ - and $1 \beta$-hydroxy- $3 \alpha-\left(4^{\prime}\right.$-oxoundec- $\left(5^{\prime} E\right)$ enyl)-pyrrolizidines were synthesized by Snider and Gao [7], and all of the spectral data were identical to those of epohelmins A and B. Revised NMR assignments for epohelmins, which were isolated as the acetate salts, are shown in Table 1. We thus here report the revised structures of epohelmins A and B, $1 \alpha$-hydroxy- $3 \alpha$-( $4^{\prime}$-oxoundec$\left(5^{\prime} E\right)$-enyl)-pyrrolizidine and $1 \beta$-hydroxy- $3 \alpha-\left(4^{\prime}-\right.$ oxoundec-(5'E)-enyl)-pyrrolizidine, respectively, and their absolute configurations as $(1 R, 3 S, 7 \mathrm{a} S)$ and $(1 S, 3 S, 7 \mathrm{a} S)$, respectively.

\section{References}

1. Sakano Y, Shibuya M, Matsumoto A, Takahashi Y, Tomoda $\mathrm{H}$, Ōmura S, Ebizuka Y. Lanopylins $\mathrm{A}_{1}, \mathrm{~B}_{1}, \mathrm{~A}_{2}$ and $\mathrm{B}_{2}$, novel lanosterol synthase inhibitors from Streptomyces sp. K995041. J Antibiot 56: 817-826 (2003)

2. Sakano Y, Shibuya M, Yamaguchi Y, Masuma R, Tomoda H, Ōmura S, Ebizuka Y. Epohelmins A and B, novel lanosterol synthase inhibitors from a fungal strain FKI-0929. J Antibiot 57: 564-568 (2004)

3. Snider BB, Zhou J. Synthesis of lanopylin $\mathrm{B}_{1}$. J Org Chem 70: 1087-1088 (2005) 
4. Glass RS, Deardorff DR, Gains LH. Pyrrolizidine synthesis by intramolecular cyclization of a substituted azacyclooctane-4,5-oxide. Tetrahedron Lett 33: 2965-2968 (1978)

5. Subramanian T, Lin CC, Lin CC. Synthesis of oxazolidinyl azacycles via ring-closing olefin metathesis: a practical entry to the synthesis of deoxy-azasugars and hydroxypyrrolizidines. Tetrahedron Lett 42: 4079-4082 (2001)

6. Poon THW, Pringle K, Foote CS. Reaction of cyclooctenes with singlet oxygen. Trapping of a perepoxide intermediate. J Am Chem Soc 117: 7611-7618 (1995)

7. Snider BB, Gao X. Structure revision and syntheses of epohelmins A and B. Org Lett 7: 4419-4422 (2005) 